\title{
Minimizing Data and Synchronization Costs in One-Way Communication
}

\author{
M. Kandemir ${ }^{*+}$ \\ N. Shenoy ${ }^{\ddagger \dagger}$ \\ P. Banerjee ${ }^{\ddagger}$ \\ J. Ramanujam \\ A. Choudhary
}

\begin{abstract}
In contrast to the conventional send/receive model, the one-way communication model-using Put and Synch-allows the decoupling of message transmission from synchronization. This opens up new opportunities not only to further optimize communication but also to reduce synchronization overhead. In this paper, we present a general technique which uses a global dataflow framework to optimize communication and synchronization in the context of the oneway communication model. Our approach works with the most general data alignments and distributions in languages like HPF, and is more powerful than other current solutions for eliminating redundant synchronization messages. Preliminary results on several scientific benchmarks demonstrate that our approach is successful in minimizing the number of data and synchronization messages.
\end{abstract}

\section{Introduction}

Most of the current compilers for distributed memory machines rely on the send and recv primitives to implement communication. The impact of this approach is twofold. First, this technique combines synchronization with communication in the sense that data messages also carry implicit synchronization information. While this relieves the compiler of the job of inserting synchronization messages to maintain data integrity, separating synchronization messages from data messages may actually improve the performance of some programs. Second, the compiler has the difficult task of matching send and recv operations in order to guarantee correct execution.

In this paper, we focus on compilation of programs annotated by HPF-like directives with one-way communication operations Put and Synch, introduced by Gupta and Schonberg [6] and Hinrichs [8]. Let us consider Figure 1(a); here, a consumer processor sends a Synch message to the producer informing that the producer can put data in a buffer physically located in the consumer's memory. After receiving the Synch message, the producer deposits the data in that buffer. We note that the Synch operation is necessary for the repetition of this communication; that is, when the producer wants to deposit new data into the buffer, it must know that the consumer has indeed consumed the old data in the buffer.

After briefly discussing the fundamental concepts used in this paper in Section 2, in Section 3 we show how the communica-

\footnotetext{
*Elec. Engr. \& Comp. Sci. Dept., Syracuse University, Syracuse, NY 13244 e-mail: mtk@ece.nwu.edu

${ }^{\dagger}$ Supported in part by NSF Young Investigator Award CCR-9357840 and NSF grant CCR-9509143

${ }^{\ddagger}$ Elec. \& Comp. Engr. Dept., Northwestern University, Evanston, IL 60208. email: \{nagaraj, banerjee, choudhar\}@ece.nwu.edu

${ }^{\S}$ Supported in part by NSF under grant CCR-9526325 and in part by DARPA under contract DABT-63-97-C-0035.

ฯ Elec. \& Comp. Engr. Dept., Louisiana State University, Baton Rouge, LA 70803. e-mail: jxr@ee.lsu .edu. Supported in part by NSF Young Investigator Award CCR9457768 and NSF grant CCR-9210422.
}

tion sets as well as the producers and the consumers manipulated by the Put operation can be implemented on top of the existing send/recv type of communication framework in our compiler [3]. Having determined those, the next issue is to minimize the number of Put communications as well as the communication volume. Section 4 presents an algorithm to achieve this goal. Our algorithm can take arbitrary control flow (excluding goto statements) into account and can optimize programs with all types of HPF-like alignments and distributions, including block-cyclic distributions. It is based on a linear algebra framework introduced by Ancourt et al. [2]; in addition, our approach is quite general in the sense that several current solutions to the problem can be derived by a suitable definition of associated predicates.

Clearly, in a compilation framework based on the Put operation, the correct ordering of memory accesses has to be imposed by the compiler using the synchronization primitives. A straightforward approach inserts a Synch operation just before each Put operation as shown in Figure 1(a). The next question to be addressed then is whether or not every Synch operation inserted that way is always necessary. The answer is no, and Section 5 proposes an algorithm to eliminate redundant synchronization messages. We refer to a Synch operation as redundant if its functionality can be fulfilled by other data communications or other Synch operations occurring in the program. The basic idea is to use another message in the reverse direction between the same pair of processors in place of the Synch call as shown in Figure 1(b). In such a situation, we say that the communication $\mathrm{t}_{j}$ kills the synchronization requirement of communication $i$. We show that our algorithm is very fast and more powerful than the previous work in synchronization elimination. This is because (1) it is very accurate in eliminating redundant synchronization, since it works at the granularity of a processor-pair using the Omega library [11]; (2) it can eliminate a synchronization message by using several data messages; (3) it handles block, cyclic, and block-cyclic distributions in a unified manner, whereas the previous approaches either work on virtual processor grids only or use an extension of regular section descriptors which are inherently inaccurate; and (4) it is preceded by a global communication optimization algorithm which itself eliminates a lot of synchronization messages. To show the idea behind the algorithm, we consider Figure 2(a), where eight processors (numbered 0 thru 7) are involved in a Put communication that repeats itself (as in a loop); processor $i$ deposits data in the memory of processor $i-1$ for $1 \leq i \leq 7$; the arrows indicate the direction of communication. Figure 2(b) shows the Synch messages required for the repetitions of this communication. Suppose that between successive repetitions of the communication pattern in Figure 2(a), subsets of processors are involved in communication patterns using Put shown in Figures 2(c) and 2(d). Our synchronization elimination algorithm can detect that the communications given in Figures 2(c) and 2(d), together, kill the synchronization requirement of the first communication, i.e., kill the Synch messages shown in Figure 2(b).

In Section 6, we give preliminary results on several benchmark 


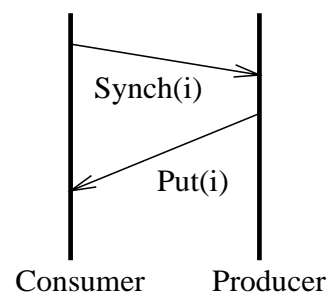

(a)

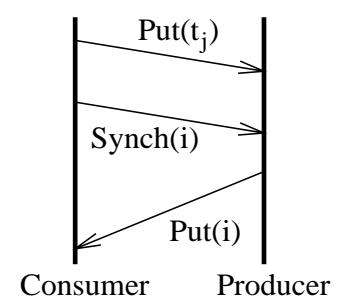

(b)

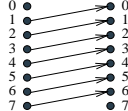

(a)

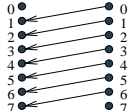

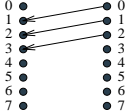

(c)

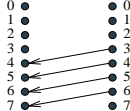

Figure 2: Communication and synchronization messages for the first loop of the program in Figure 6(a).

Figure 1: (a) one-way communication with Put operation. (b) elimination of a Synch message.

programs. Our experiments show that we are able to reduce data messages on the average by $37 \%$ and synchronization messages by $96 \%$. We believe that these are also the first results from a comprehensive evaluation of synchronization elimination in one-way communication. We discuss related work in Section 7 and conclude the paper with a summary in Section 8 .

\section{Preliminaries}

We focus on structured programs with conditional statements and nested loops but without arbitrary goto statements. A basic block is a sequence of consecutive statements in which the flow of control enters at the beginning and leaves at the end without the possibility of branching except perhaps at the end [1]. A control flow graph (CFG) is a directed graph constructed by basic blocks and represents the flow-of-control information of the program. For the purpose of this paper, the CFG can be thought of as a directed graph $\mathcal{G}=(\mathcal{V}, \mathcal{E})$ where each $v \in \mathcal{V}$ represents either a basic block or a (reduced) interval that represents a loop, and each $e \in \mathcal{E}$ represents an edge between blocks. In this paper, depending on the context, we use the term node interchangeably for a statement, a block or an interval. Two unique nodes $s$ and $t$ denote the start and terminal nodes respectively of a CFG. One might think of these nodes as dummy statements. We define the sets of all successors and predecessors of a node $n$ as $\operatorname{succ}(n)=\{m \mid(n, m) \in \mathcal{E}\}$ and $\operatorname{pred}(n)=\{m \mid(m, n) \in \mathcal{E}\}$, respectively. Node $i$ dominates node $j$ in the CFG (written as $j \in \operatorname{dom}(i)$ ), if every path from $s$ to $j$ goes through $i$. We assume that prior to communication analysis, any edge that goes directly from a node with more than one successor to a node with more than one predecessor is split by introducing a dummy node. Our technique for minimizing the communication volume and the number of messages is based on interval analysis [1]. Interval analysis consists of a contraction phase and an expansion phase. For programs written in a structured language, an interval corresponds to a loop. The contraction phase collects information about what is generated and what is killed inside each interval. Then the interval is reduced to a single node and annotated with the information collected. This is a recursive procedure and stops when the reduced CFG contains no more cycles. In each step of the expansion phase, a node (reduced interval) is expanded, and the information regarding the nodes in that interval is computed.

\section{Producers and Consumers}

We assume that all loop bounds and subscript expressions are affine functions of enclosing loop indices and symbolic variables. Under this condition, a loop nest, an array and a processor grid can all be represented as bounded polyhedra. Our compiler currently uses the owner-computes rule [9], which assigns each computation to the processor that owns the data being computed.

Consider the generic single loop $i$ shown in Figure 3(a). Let $\mathcal{R}_{\mathcal{L}}(i)=\mathrm{X}\left(\gamma_{L} * i+\theta_{L}\right)$ and $\mathcal{R}_{\mathcal{R}}(i)=\mathrm{Y}\left(\gamma_{R} * i+\theta_{R}\right)$. Let $p$ and $q$ denote two processors. We define several sets shown in Figure 3(b) where $\mathrm{S}$ is the communication statement and $\vee$ and $\wedge$ are logical 'or' and 'and' operations respectively. The set $0 \mathrm{wn}(\mathrm{X}, p)$ refers to the elements of array $\mathrm{X}$ mapped onto processor $p$ through compiler directives. Similar 0wn sets are defined for other arrays as well. The sets Producers (S) and Consumers (S) denote, respectively, the processors that produce and consume data communicated in S. For a specific processor, ProducersFor and ConsumersFor give the set of processors that send data to and receive data from that processor respectively. PutSet $(\mathrm{S}, p, q)$ is the set of elements that should be put (written) by processor $q$ to memory of processor $p$. SendSet (S) is set of pairs $\left(q^{\prime}, p^{\prime}\right)$ such that $q^{\prime}$ sends data to (write data in the memory of) $p^{\prime}$. Finally, Pending (S) is the inverse of SendSet (S), and gives set of pairs $\left(p^{\prime}, q^{\prime}\right)$ such that $p^{\prime}$ should send a Synch message to $q^{\prime}$ for the repetitions of the communication (in each iteration of the time-step loop $t$ ) occurring in S. For a communication occurring in $i$, the set Pending ( $i$ ) represents a list of individual Synch messages that should be sent for the safe repetition of the data communication in $i$. That is, a Synch message is between just a pair of processors. For an $i$ and a Synch, we say whether or not Synch $\in$ Pending(i).

In fact, by using appropriate projection functions all of those sets can be obtained from a single set called Commset(S) containing triples $\left(p^{\prime}, q^{\prime}, d\right)$ meaning that element $d$ should be communicated from $q^{\prime}$ to $p^{\prime}$ in $\mathrm{S}$. The CommSet (S) is currently used in our compiler's communication generation portion to generate send and recv commands. The necessary projection functions can be implemented by using the Omega library [11], and are shown in Figure 3(c). For instance, ConsumersFor $(\mathrm{S}, q)$ is obtained from Commset (S) by projecting out $d$ and substituting $q$ for $q^{\prime}$; that is, $q$ is a parameter and ConsumersFor $(\mathrm{S}, q)$ enumerates $p^{\prime}$ values in terms of $q$.

By taking into account the alignment and distribution information, we can define the $0 \mathrm{wn}$ set more formally as

$$
\begin{aligned}
\operatorname{Own}(\mathrm{Y}, q) & =\{d \mid \exists t, c, l \text { s. t. }(t=\alpha d+\beta=\mathcal{C} P c+\mathcal{C} q+l) \\
& \wedge\left(y_{l} \leq d \leq y_{u}\right) \wedge\left(p_{l} \leq q \leq p_{u}\right) \wedge\left(t_{l} \leq t \leq t_{u}\right) \\
& \wedge(0 \leq l \leq \mathcal{C}-1)\}
\end{aligned}
$$

where $P=p_{u}-p_{l}+1$. In this formulation, $t=\alpha d+\beta$ represents alignment information and $t=\mathcal{C} P c+\mathcal{C} q+l$ denotes distribution information. In other words, each array element $d$ is mapped onto a point in a two-dimensional array. This point can be represented by a 


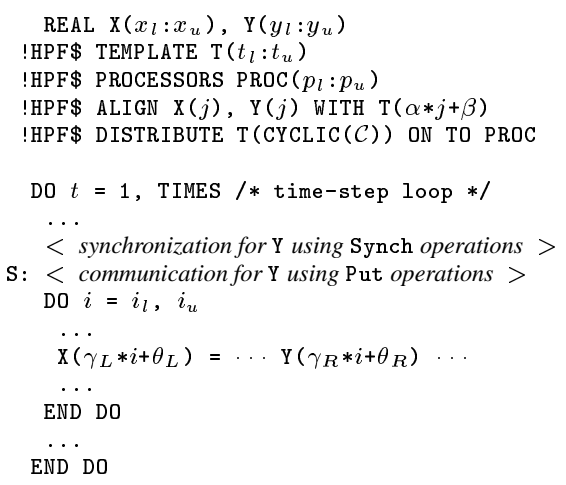

\begin{tabular}{|c||l|}
\hline SET & PROJECTION FUNCTION \\
\hline \hline Producers (S) & {$\left[p^{\prime}, q^{\prime}, d\right] \mapsto\left[q^{\prime}\right]$} \\
\hline Consumers (S) & {$\left[p^{\prime}, q^{\prime}, d\right] \mapsto\left[p^{\prime}\right]$} \\
\hline ProducersFor(S, $p)$ & {$\left[p, q^{\prime}, d\right] \mapsto\left[q^{\prime}\right]$} \\
\hline ConsumersFor $(\mathbf{S}, q)$ & {$\left[p^{\prime}, q, d\right] \mapsto\left[p^{\prime}\right]$} \\
\hline PutSet $(\mathrm{S}, p, q)$ & {$[p, q, d] \mapsto[d]$} \\
\hline SendSet(S) & {$\left[p^{\prime}, q^{\prime}, d\right] \mapsto\left[q^{\prime}, p^{\prime}\right]$} \\
\hline Pending (S) & {$\left[p^{\prime}, q^{\prime}, d\right] \mapsto\left[p^{\prime}, q^{\prime}\right]$} \\
\hline
\end{tabular}

(c)

(a)

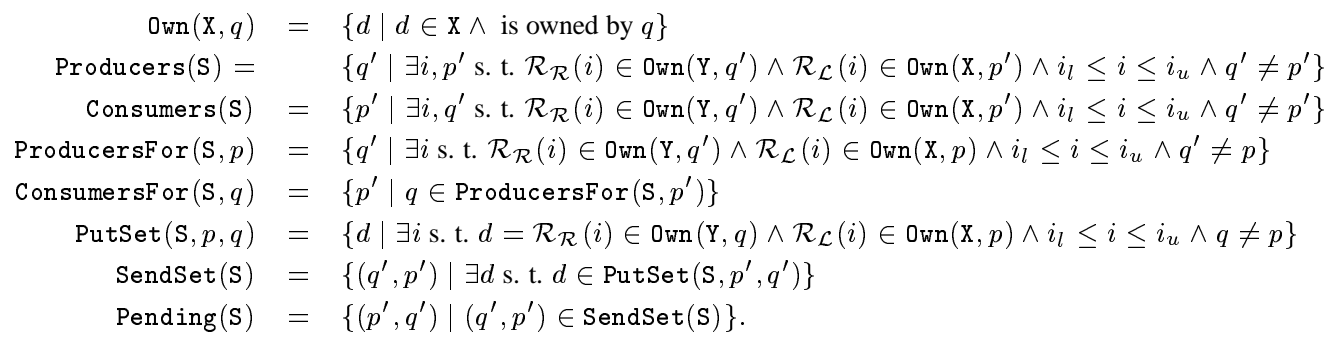

(b)

Figure 3: (a) Generic loop. (b) Several sets. (c) Projection functions to manipulate sets ( $p$ and $q$ are symbolic names).

pair $(c, l)$ and gives the local address of the data item in a processor. Simple BLOCK and CYCLIC(1) distributions can be handled within this framework by setting $c=0$ and $l=0$, respectively. The formulation given here can be generalized to multi-dimensional loops, arrays and processor grids [2]. Consider the first i-loop in Figure 6(b). Figure 4(a) shows the sets for this loop assuming that in the transformed program array bounds start from 0 . Notice that a processor $q$ is in the Producers set if there exists a processor $p$ such that $q \neq p$ and $q$ puts data in $p$ 's memory. Similarly, a processor $p$ is in the Consumers set if there exists a processor $q$ such that $p \neq q$ and $q$ puts data in $p$ 's memory. For this example, if distribution directive for the arrays is changed to CYCLIC (4), then we have the sets shown in Figure 4(b). All of these sets can easily be represented and manipulated by the Omega library [11]. Notice that using the Omega sets to represent producer-consumer information, we are able to accommodate any kind of HPF-like alignment and distribution data in our framework through 0wn sets.

Let $\{\vec{d} \mid \mathcal{P}(\vec{d})\}$ and $\{\vec{d} \mid \mathcal{Q}(\vec{d})\}$ be two PutSets for a same multi-dimensional array where $\mathcal{P}($.$) and \mathcal{Q}($.) denote two predicates and $\vec{d}$ refers to an array element. We define three operations, $\vee_{c},-{ }_{c}$, and $\Lambda_{c}$ on these PutSets as shown in Figure 4(c). In the remainder of this paper, $\bigvee$ and $\bigwedge$ symbols will also be used for $\bigvee_{c}$ and $\wedge_{c}$, respectively, when there is no confusion.

\section{Optimizing Communication}

The objective of our global communication optimization framework is to determine PutSet $(i, p, q)$ for each node $i$ in the program globally; that is, by taking into account all the nodes in the CFG that are involved in communication.
Local (Intra-Interval) Analysis The local analysis part of our framework computes Kill, Gen and Post_Gen sets defined below for each interval. Then the interval is reduced to a single node and annotated with these sets. With reference to Figure 3(a),

$$
\begin{aligned}
\operatorname{Kill}(\mathrm{i}, q)= & \{(\vec{d} \mid \vec{d} \in \operatorname{Own}(\mathrm{X}, q)) \wedge(\exists \vec{\imath} \text { s. t. } \\
& \left.\left.\left(\vec{d}=\mathcal{R}_{\mathcal{L}}(\vec{\imath})\right) \wedge\left(\overrightarrow{i_{l}} \leq \vec{\imath} \leq \overrightarrow{i_{u}}\right)\right)\right\}, \\
\operatorname{Modified}(\mathrm{i}, q)= & {\left[\bigvee_{j \in \operatorname{pred}(i)} \operatorname{Modified}(j, q)\right] \vee_{c} \operatorname{Kill}(\mathrm{i}, q) . }
\end{aligned}
$$

assuming Modified $(\operatorname{pred}(\operatorname{first}(i)), q)=\emptyset$ where $\operatorname{first}(i)$ is the first node in $i$.

$\operatorname{Kill}(\mathbf{i}, q)$ is the set of elements owned and written (killed) by processor $q$ locally in $\mathbf{i}$, and Modified $(\mathbf{i}, q)$ is the set of elements that may be killed along any path from the beginning of the interval to (and including) $i$. The computation of the Kill set proceeds in the forward direction; that is, the nodes within the interval are traversed in topological sort order. If last (i) is the last node in $i$, then

$$
\operatorname{Kill}(\mathbf{i}, q)=\operatorname{Modified}(\operatorname{last}(\mathbf{i}), q)
$$

This last equation is used to reduce an interval into a node. The reduced interval is then annotated by its Kill set.

$\operatorname{Gen}(\mathbf{i}, p, q)$ is the set of elements to be written by $q$ into $p$ 's memory to satisfy the communication in $i$. The computation of the Gen proceeds in the backward direction, i.e., the nodes within each interval are traversed in reverse topological sort order. The elements that can be written by $q$ into $p$ 's memory at the beginning of a node in the CFG are the elements required by $p$ due to a RHS reference in the node except the ones that are written locally (killed) by $q$ before being referenced by $p$. 


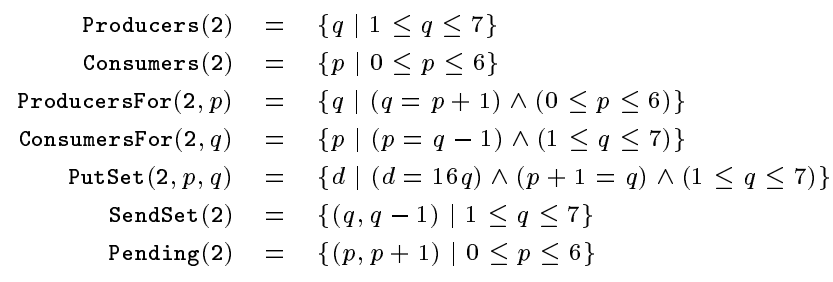

(a)

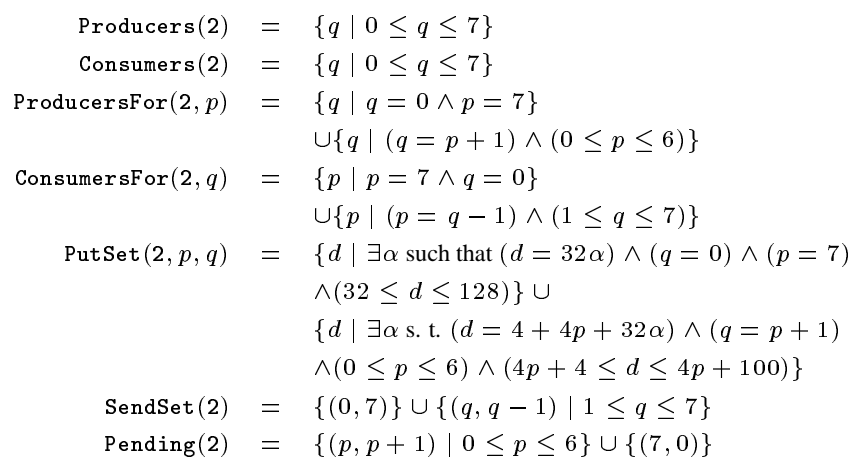

$$
\begin{aligned}
\{\vec{d} \mid \mathcal{P}(\vec{d})\} \vee_{c}\{\vec{d} \mid \mathcal{Q}(\vec{d})\} & =\{\vec{d} \mid \mathcal{P}(\vec{d}) \vee \mathcal{Q}(\vec{d})\} \\
\{\vec{d} \mid \mathcal{P}(\vec{d})\}-_{c}\{\vec{d} \mid \mathcal{Q}(\vec{d})\} & =\{\vec{d} \mid \mathcal{P}(\vec{d}) \wedge \neg(\mathcal{Q}(\vec{d}))\} \\
\{\vec{d} \mid \mathcal{P}(\vec{d})\} \wedge_{c}\{\vec{d} \mid \mathcal{Q}(\vec{d})\} & =\{\vec{d} \mid \mathcal{P}(\vec{d}) \wedge \mathcal{Q}(\vec{d})\}
\end{aligned}
$$

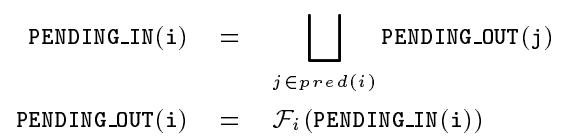

(d)

Figure 4: (a) Sets for the first loop in Figure 6(b) for BLOCK distribution. (b) Sets for the first loop in Figure 6(b) for CYCLIC(4) distribution. (c) Operations on PutSets. (d) Dataflow equations for optimizing synchronization messages.

Assuming $\vec{\imath}=\left(\imath_{1}, \ldots, \imath_{n}\right)$ and $\vec{\imath}=\left(\imath_{1}^{\prime}, \ldots, \imath_{n}^{\prime}\right)$, let $\vec{\imath} \prec \vec{\imath}$ mean that $\overrightarrow{\imath^{\prime}}$ is lexicographically less than or equal to $\vec{\imath}$; and $\vec{\imath} \prec_{k} \vec{\imath}$ mean that $\imath_{j}^{\prime}=\imath_{j}$ for all $j<k$, and $\left(\imath_{k}^{\prime}, \ldots, \imath_{n}^{\prime}\right) \prec\left(\iota_{k}, \ldots, \imath_{n}\right)$. Let $\operatorname{Comm}(i, p, q)$ be the set of elements that may be communicated at the beginning of interval $i$ to satisfy communication requirements from the beginning of $i$ to the last node of $i$. Then, from Figure 3(a), assuming that $\operatorname{Comm}(\operatorname{succ}(\operatorname{last}(i)), q)=\emptyset$, we have

$$
\begin{aligned}
\operatorname{Gen}(\mathbf{i}, p, q)= & \left\{\vec{d} \mid \exists \vec{\imath} \text { s. t. }\left(\overrightarrow{i_{l}} \leq \vec{\imath} \leq \overrightarrow{i_{u}}\right) \wedge\right. \\
& \left(\vec{d}=\mathcal{R}_{\mathcal{R}}(\vec{\imath}) \in 0 \mathrm{wn}(\mathrm{Y}, q)\right) \wedge\left(\mathcal{R}_{\mathcal{L}}(\vec{\imath}) \in \operatorname{Own}(\mathrm{X}, p)\right) \\
& \wedge \neg\left(\exists \vec{\jmath}, \mathcal{R}_{\mathcal{L}}{ }^{\prime} \text { s. t. }\left(\overrightarrow{i_{l}} \leq \vec{\jmath} \leq \overrightarrow{i_{u}}\right) \wedge\left(\vec{d}=\mathcal{R}_{\mathcal{L}}(\vec{\jmath})\right)\right. \\
& \left.\left.\wedge\left(\vec{\jmath} \prec_{\text {level }(\mathbf{i})} \vec{\imath}\right)\right)\right\}, \\
\operatorname{Comm}(\mathbf{i}, p, q)= & {\left[\bigwedge_{s \in \operatorname{succ}(i)} \operatorname{Comm}(\mathrm{s}, \mathrm{p}, \mathrm{q})\right] \vee_{c} \operatorname{Gen}(\mathrm{i}, \mathrm{p}, \mathrm{q}) . }
\end{aligned}
$$

The negated condition eliminates all the elements written by $q$ locally in an earlier iteration than the one in which $p$ requires them. In addition, we use the following equation to reduce an interval into a single node

$$
\operatorname{Gen}(i, p, q)=\operatorname{Comm}(\operatorname{First}(\mathbf{i}), p, q) .
$$

In the definition of Gen, $\mathcal{R}_{\mathcal{R}}$ denotes the RHS reference, and $\mathcal{R}_{\mathcal{L}}$ denotes the LHS reference of the same statement. $\mathcal{R}_{\mathcal{L}}{ }^{\prime}$, on the other hand, refers to any LHS reference within the same interval. Notice that while $\mathcal{R}_{\mathcal{L}}{ }^{\prime}$ is a reference to the same array as $\mathcal{R}_{\mathcal{R}}$, in general $\mathcal{R}_{\mathcal{L}}$ can be a reference to any array. level(i) gives the nesting level of the interval (loop), 1 corresponding to the outermost loop in the nest.

After the interval is reduced, the Gen set for it is recorded, and an operator $\mathcal{N}$ is applied to the last part of this Gen set to propagate it to the outer interval:

$$
\mathcal{N}\left(\vec{\jmath} \prec_{\mathrm{k}} \vec{\imath}\right)=\vec{\jmath} \prec_{(\mathrm{k}-1)} \vec{\imath} .
$$

It should be emphasized that computation of Gen sets gives us all the communication that can be vectorized above a loop nest; that is, our analysis easily handles message vectorization [9]. A naive implementation may set Put_Set $(\mathbf{i}, p, q)$ to $\operatorname{Gen}(\mathbf{i}, p, q)$ for every $i, p$ and $q$. But such an approach often retains redundant communication which would otherwise be eliminated.

Finally, Post_Gen $(i, p, q)$ is the set of elements to be written by $q$ into memory of $p$ at node $i$ with no subsequent local write to them by $q$ :

$$
\begin{aligned}
\text { Post_Gen }(i, p, q)= & \left\{\vec{d} \mid \exists \vec{\imath} \text { s. t. }\left(\overrightarrow{i_{l}} \leq \vec{\imath} \leq \overrightarrow{i_{u}}\right) \wedge\right. \\
& \left(\vec{d}=\mathcal{R}_{\mathcal{R}}(\vec{\imath}) \in 0 \operatorname{wn}(\mathrm{Y}, q)\right) \wedge\left(\mathcal{R}_{\mathcal{L}}(\vec{\imath}) \in 0 \operatorname{wn}(\mathrm{X}, p)\right) \\
& \wedge \neg\left(\exists \vec{\jmath}, \mathcal{R}_{\mathcal{L}}^{\prime} \text { s. t. }\left(\overrightarrow{i_{l}} \leq \vec{\jmath} \leq \overrightarrow{i_{u}}\right) \wedge\left(\vec{d}=\mathcal{R}_{\mathcal{L}}{ }^{\prime}(\vec{\jmath})\right)\right. \\
& \left.\left.\wedge\left(\vec{\imath} \prec_{\text {level }(\mathrm{i})} \vec{\jmath}\right)\right)\right\} .
\end{aligned}
$$

The computation of Post_Gen $(\mathbf{i}, p, q)$ proceeds in the forward direction. Its computation is very similar to those of Kill and Gen sets, so we do not discuss it further.

Dataflow Equations In our framework, one-way communication calls are placed at the beginning of nodes in the CFG. Our dataflow analysis consists of a backward and a forward pass. In the backward pass, the compiler determines sets of data elements that can safely be communicated at specific points. The forward pass, on the other hand, eliminates redundant communication and determines the final set of elements that should be communicated (written by $q$ into $p$ 's memory) at the beginning of each node $i$. The 
input for the equations consists of the Gen, Kill and Post_Gen sets.

The dataflow equations for the backward analysis are given by Equations (1) and (2) in Figure 5. Basically, they are used to combine and hoist communication. The sets $\operatorname{Safe} \operatorname{In}(\mathbf{i}, p, q)$ and Safe_Out $(i, p, q)$ consist of elements that can safely be communicated at the beginning and end of node $i$, respectively. Equation (1) says that an element should be communicated at a point if and only if it will be used in all of the following paths in the CFG. Equation (2), on the other hand, gives the set of elements that can safely be communicated at the beginning of $i$. Intuitively, an element can be written by $q$ into $p$ 's memory at the beginning of $i$ if and only if it is either required by $p$ in $i$ or it reaches at the end of $i$ (in the backward analysis) and is not overwritten (killed) by the owner $(q)$ in it. The predicate $\mathcal{P}(\mathbf{i})$ is used to control communication hoisting. If $\mathcal{P}(i)$ is true, communication is not hoisted to the beginning of $i . \mathcal{P}(\mathbf{i})=$ false implies aggressive communication combining and hoisting. An algorithm can also put a condition which tests the compatibility between $\operatorname{Gen}(i, p, q)$ and Safe_Out $(i, p, q)$ (e.g. two left-shift communications are compatible whereas a left-shift and a right-shift are not) [4].

The task of the forward analysis phase, which makes use of Equations (3), (4) and (5) in Figure 5, is to eliminate redundant communication by observing that (1) a node in the CFG should not have a non-local datum which is exclusively needed by a successor unless it dominates that successor; and (2) a successor should ignore what a predecessor has so far unless that predecessor dominates it. Put_In $(i, p, q)$ and Put_Out $(i, p, q)$ denote the set of elements that have been written so far (at the beginning and end of node $i$ respectively) by $q$ into memory of $p$. Equation (3) conservatively says that the communication set arriving in a join node can be found by intersecting the sets for all the joining paths. Equation (4) is used to compute the PutSet set which corresponds to the elements that can be communicated at the beginning of the node except the ones that have already been communicated (Put_In). The elements that have been communicated at the end of node $i$ (that is, Put_Out set) are simply the union of the elements communicated up to the beginning of $i$ (that is, Put_In set), the elements communicated at the beginning of $i$ (that is, PutSet ( $i, p, q)$ set) (except the ones which have been killed in $i$ ) and the elements communicated in $i$ and not killed subsequently (Post_Gen).

Interval Analysis Our approach starts by computing the Gen, Kill and Post_Gen sets for each node. Then the contraction phase reduces the intervals from the innermost loop to the outermost loop and annotates them with Gen, Kill and Post_Gen sets. When a reduced CFG with no cycles is reached, the expansion phase starts and PutSets for each node is computed from the outermost loop to the innermost loop. There is one important point to note: before starting to process the next graph in the expansion phase, the Put_In set of the first node in this graph is set to the PutSet of the interval that contains it to avoid redundant communication. More formally, in the expansion phase, we set Put_In $(i, p, q)^{k^{t h}}$ pass $=$

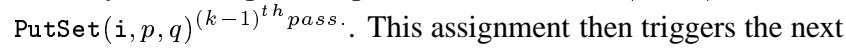
pass in the expansion phase. Before the expansion phase starts Put_In $(\mathbf{i}, \mathbf{p}, \mathbf{q})^{1^{\text {st }}}$ pass is set to the empty set. Note that the whole dataflow procedure operates on sets of equalities and inequalities which can be manipulated by the Omega library [11] or a similar tool.

Example Consider the synthetic benchmark given in Figure 6(a). In this example, communication occurs for three arrays: B, D and F. A communication optimization scheme based on message vector- ization alone, can place communications and associated synchronizations as shown in Figure 6(b) before the loop bounds reduction. Note that a Synch message in that figure in fact represents a number of point-to-point synchronization messages. An application of our global communication optimization method generates the program shown in Figure 6(c). As compared with the message vectorized version, there is a $50 \%$ reduction (from 28 to 14) in the number of messages and $40 \%$ in the communication volume (from 35 to 21) across all processors. We note that we can optimize this program even the distribution directive is changed to CYCLIC $(\mathrm{K})$ for any $\mathrm{K}$. When the distribution directive is CYCLIC (4), we have a $50 \%$ reduction (from 48 to 24 ) in the number of messages and $32 \%$ reduction (from 139 to 94 ) in the communication volume across all processors. Note that our approach here reduces the original number of synchronization messages as well (from 28 to 14 for the BLOCK distribution and from 48 to 24 for the CYCLIC(4) case).

\section{Optimizing Synchronization}

In this section, we assume that the compiler has conducted the dataflow analysis described in Section 4 and determined the optimal communication points and communication sets. Assuming that these communications will be implemented by Put operations, we present a dataflow analysis to minimize the number of Synch messages. We assume that communication patterns (i.e. producerconsumer relationships) are identical for each repetition of a communication. For example, in Figure 6(c), the producer-consumer pattern for the communication occurring in 1 is identical for every repetition of time-step loop $t$.

Our approach first makes a single pass over the current interval and determines some synchronizations that cannot be eliminated by the analysis to be described. We call the set of synchronizations (associated with a node $i$ ) that cannot be eliminated SynchFix (i). We refer the reader to [10] for the definition of SynchFix ( $i$ ).

The dataflow technique described here starts with the deepest loops and works its way through loops in a bottom up manner, handling one loop at a time. It then reduces the loop to a node and annotates it with its final synchronization requirements that cannot be eliminated. The procedure works on an augmented CFG, where each communication loop is represented by a single node. In the following, the symbol $i$ refers to such a node.

For a given i, we can define the set SynchSet as a set of processor pairs that should be synchronized after our analysis. In a straightforward implementation, SynchSet $(i)=\operatorname{Pending}(i)$ for each $i$. We would like to reduce the cardinality of SynchSet (i) for each $i$. We say that no synchronization is required for a communication $i$, if SynchSet (i) happens to be empty after our dataflow analysis.

If the compiler wants to eliminate a Synch message for communication $i$ from $p$ to $q$, it needs to find a message $\mathrm{t}_{j}$ for another communication from $p$ to $q$ and use it as synchronization. Such a message should occur between repetitions of $i$ and after the data value communicated at $i$ is consumed. Suppose that a specific producer $q$ and a consumer $p$ are involved in a communication in $i$. Consider all $k$ communications $\mathrm{t}_{j}(1 \leq j \leq k)$ occurring after the value communicated in $i$ is consumed by $p$ and before the next repetition of $i$. Then, if the following holds, the Synch message from $p$ to $q$ can be eliminated:

$$
\left(\exists j \mid(1 \leq j \leq k) \wedge q \in \text { ConsumersFor }\left(\mathrm{t}_{j}, p\right)\right)
$$

An interesting case occurs when all the Synch messages contained in the Pending( $i$ ) set for a specific $i$ are eliminated. We can formalize this condition as

$$
\forall p \forall q\left(((p, q) \in \operatorname{Pending}(\mathrm{i})) \quad \Rightarrow \quad \exists j\left((p, q) \in \operatorname{SendSet}\left(\mathrm{t}_{j}\right)\right)\right) \text { (6) }
$$


Backward Analysis:

$$
\begin{aligned}
& \text { Safe_Out }(i, p, q)=\bigwedge_{s \in \operatorname{succ}(i)} \operatorname{Safe\_ In}(\mathbf{s}, p, q) \\
& \text { Safe_In }(\mathbf{i}, p, q)= \begin{cases}\operatorname{Gen}(\mathbf{i}, p, q) & \text { if } \mathcal{P}(\mathbf{i}) \\
\left(\operatorname{Safe\_ Out}(\mathbf{i}, p, q)-{ }_{c} \operatorname{Kill}(\mathbf{i}, q)\right) \vee_{c} \operatorname{Gen}(\mathbf{i}, p, q) & \text { otherwise }\end{cases}
\end{aligned}
$$

Forward Analysis:

$$
\begin{aligned}
& \text { Put_In }(\mathrm{i}, p, q)=\bigwedge_{j \in \text { pred }(i)} \text { Put_Out }(\mathrm{j}, p, q) \\
& \operatorname{PutSet}(\mathbf{i}, p, q)= \begin{cases}\operatorname{Gen}(\mathbf{i}, p, q)-{ }_{c} \operatorname{Put} \_\operatorname{In}(\mathbf{i}, p, q) & \text { if } \exists k \text { s.t. } k \in \operatorname{succ}(i) \text { and } k \notin \operatorname{dom}(i) \\
\operatorname{Safe\_ In}(\mathbf{i}, p, q)-{ }_{c} \operatorname{Put} \_I n(i, p, q) & \text { otherwise }\end{cases} \\
& \text { Put_Out }(\mathbf{i}, p, q)=\left\{\begin{array}{l}
\operatorname{Put} \operatorname{In}(\mathbf{i}, p, q)-{ }_{c} \operatorname{Kill}(\mathbf{i}, q) \quad \text { if } \exists k \text { s. t. } k \in \operatorname{succ}(i) \text { and } k \notin \operatorname{dom}(i) \\
\left(\left(\operatorname{PutSet}(\mathbf{i}, p, q)+{ }_{c} \operatorname{Put} \operatorname{In}(\mathbf{i}, p, q)\right)-{ }_{c} \operatorname{Kill}(\mathbf{i}, q)\right)+{ }_{c} \operatorname{Post} \_\operatorname{Gen}(\mathbf{i}, p, q) \quad \text { otherwise }
\end{array}\right.
\end{aligned}
$$

Figure 5: Dataflow equations for optimizing communication.

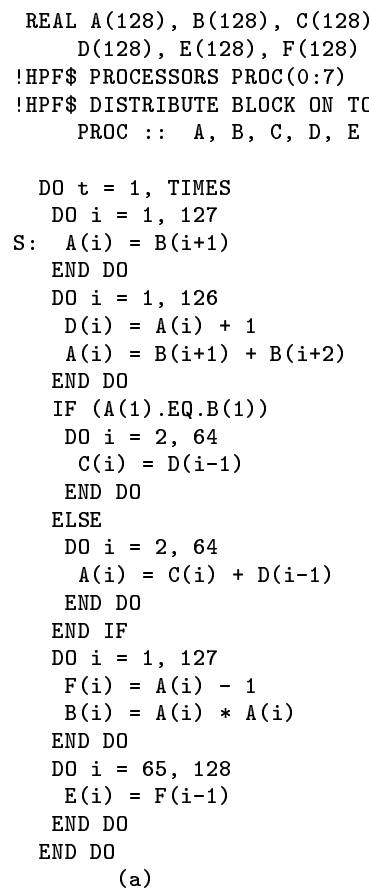

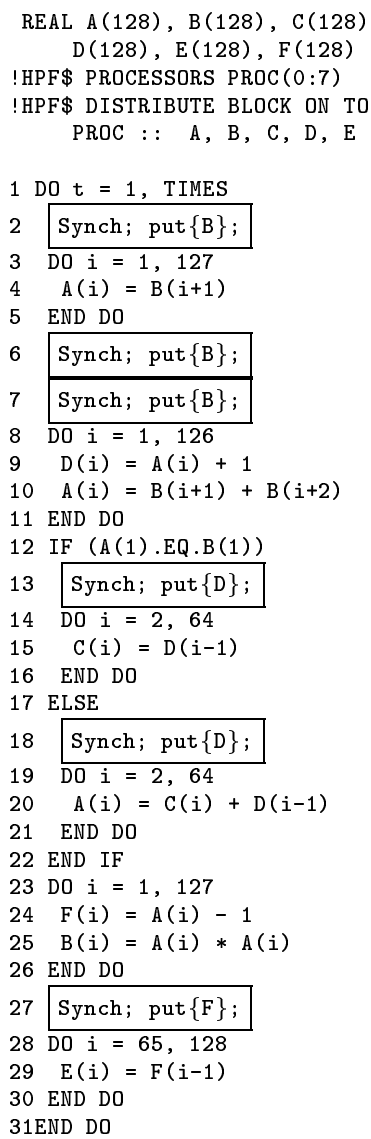

(b)
REAL A(128), B(128), C(128)

$D(128), E(128), F(128)$ ! HPF\$ PROCESSORS PROC $(0: 7)$ !HPF\$ DISTRIBUTE BLOCK ON TO PROC : : A, B, C, D, E

DO $t=1$, TIMES

1: Synch; put $\{B\}$;

DO $i=1,127$

$A(i)=B(i+1)$

END DO

DO $i=1,126$

$D(i)=A(i)+1$

$A(i)=B(i+1)+B(i+2)$

END DO

2: Synch; $\operatorname{put}\{\mathrm{D}\}$;

IF (A(1).EQ.B(1))

DO $i=2,64$

$C(i)=D(i-1)$

END DO

ELSE

DO $i=2,64$

$A(i)=C(i)+D(i-1)$

END DO

END IF

DO $i=1,127$

$F(i)=A(i)-1$

$B(i)=A(i) * A(i)$

END DO

3: Synch; put $\{\mathrm{F}\}$;

DO $i=65,128$

$E(i)=F(i-1)$

END DO

END DO

(c)

Figure 6: (a) A synthetic benchmark. (b) Message vectorized translation. (c) Global communication optimization. 
assuming $1 \leq j \leq k$. Notice that $j$ values can be different for each $q$. If we additionally stipulate that all $j$ values should be the same for all $q$ values, then we obtain

$$
\forall p \forall q \exists j\left(((p, q) \in \text { Pending }(\mathrm{i})) \quad \Rightarrow \quad\left((p, q) \in \operatorname{SendSet}\left(\mathrm{t}_{j}\right)\right)\right)(7)
$$

We note that the condition (7) implies the algorithms offered by [6] and [8].

Claim: The synchronizations eliminated by (7) are a subset of the synchronizations that can be eliminated by (6) (see [10] for the proof).

Even if a Pending (i) set cannot be totally eliminated we can reduce its cardinality by eliminating as many Synch messages as possible from it. That is, after our analysis, for every $i$,

$$
\begin{aligned}
\operatorname{SynchSet}(\mathrm{i})= & \text { Pending }(\mathrm{i})-{ }_{c}\{(p, q) \mid \exists j \text { s. t. } \\
& \left.q \in \text { ConsumersFor }\left(\mathrm{t}_{j}, p\right)\right\} \vee_{c} \operatorname{SynchFix}(\mathrm{i})
\end{aligned}
$$

As an example, let us consider the program shown in Figure 6(c). In this program, communication occurs at three points: 1, 2 and 3. A straightforward implementation inserts three (sets of) Synch operations, corresponding to 1,2 and 3 as shown in that figure. Let us now focus on the communication in 1. Figure 2(a) shows the messages sent (Put operations) for this communication. Figure 2(b), on the other hand, shows the required synchronization messages for the repetitions of this communication. Finally, Figures 2(c) and 2(d) show the communication messages in 2 and 3 respectively. Notice that, by using the condition given in (6), the synchronization requirements for 1 can be eliminated; that is, the communications occurring in 2 and 3 together kill the synchronization requirements of the communication in 1. If we consider Figure 2(c) and Figure 2(d) separately for the condition given by (7), however, none of them individually can eliminate the synchronization for 1. By a similar argument, it can be concluded that the communications in 2 and 3 do not need any synchronization either, as their synchronization requirements are killed by communication in 1.

Dataflow Analysis: Our data flow equations are shown in Figure 4(d). Our analysis consists of iterative forward passes on the CFG. We first concentrate on the second equation and explain the functionality of $\mathcal{F}_{i}$. Here PENDING_IN (i) represents the synchronization requirements of all the communications traversed so far up to the beginning of $i$. PENDING_OUT ( $i$ ) is defined analogously for the end of $i$. Assuming that $P_{k}$ is the synchronization requirement (in terms of pairs of processors) for node $\mathrm{k}$ up to $i$ in the analysis and PENDING_IN (i) $=\left\{P_{1}, \ldots, P_{i-1}, P_{i}, P_{i+1}, \ldots, P_{m}\right\}$, we can define $\mathcal{F}_{i}$ as

$\mathcal{F}_{i}($ PEND ING_IN $(\mathrm{i}))=\left\{f_{i}\left(P_{1}\right), \ldots, f_{i}\left(P_{i-1}\right), P_{i}, f_{i}\left(P_{i+1}\right), \ldots, f_{i}\left(P_{m}\right)\right\}$

where $f_{i}\left(P_{k}\right)=P_{k}-{ }_{c} \operatorname{SendSet}(\mathbf{i})$. That is, when a node $i$ is visited, the synchronization requirements for all other communications are checked to see whether or not any synchronization can be eliminated by using the communication occurring at $i$. Prior to the analysis, $P_{i}$ is set to Pending (i) for the first node. After a fixed state is reached, the PENDING_OUT set of the last node gives the synchronization requirements to be satisfied. The resulting PENDING_OUT set, which is in fact a set of sets, is then reduced to a single set and used to represent the synchronization requirements of this loop to the next upper level.

We now consider the first equation of Figure 4(d) and explain the $\bigsqcup$ operator appearing there. In the join nodes, the compiler takes a conservative approach by unioning the synchronization requirements for the same communication. Suppose that for each $j \in \operatorname{pred}(i)$, PENDING_OUT $(j)=\left\{P_{j 1}, P_{j 2}, \ldots, P_{j m}\right\}$, where $P_{j k}$ is the synchronization requirement of communication $k$ up to the end of $j$. Assuming then that the resulting PENDING_IN $(i)=$ $\left\{P_{1}, P_{2}, \ldots, P_{m}\right\}$, each $P_{l} \in$ PENDING_IN (i) can be computed as $P_{l}=\vee_{c} P_{j l}$ where $\vee_{c}$ is performed over the $j$ values. We note that this algorithm is more accurate and faster than those proposed in [8] and [6].

Claim: The dataflow procedure defined by equations given in Figure 4(d) can reach a steady state in at most after two iterations (see [10] for the proof).

Example The upper part of Table 1 shows the sets SendSet and Pending (in an open form rather than in terms of equalities and inequalities) for the program shown in Figure 6(c). Note that the sets SendSet (1) and Pending(1) are computed from the SendSet and Pending sets respectively given in Figure 4(a). The sets for 2 and 3 are computed similarly. Before the dataflow analysis starts, PENDING_IN (1) is initialized as follows:

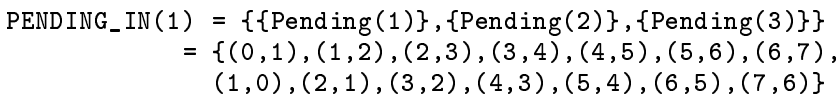

The lower part of Table 1 demonstrates application of our dataflow algorithm to this example. After the fixed state is reached, an examination of PENDING_OUT (3) reveals that the program can be executed without any synchronization.

\section{Preliminary Results}

The applications used in our study and their characteristics are listed in Table 2. We experimented with BLOCK, B-CYC (blockcyclic) and CYCLIC distributions on 8 and 32 processors to measure the static improvements. We refer to a version of program which is optimized by message vectorization alone as base. Table 3 presents the communication volume and the number of data messages (that is also the number of Synch messages) across all processors in the base programs.

For these applications, we first applied our global communication optimization algorithm. The percentage improvements are listed in Table 4. It should be noted that in block-cyclic distributions where most of the previous approaches fail, we have, on the average, a $29 \%$ reduction in communication volume and $40 \%$ reduction in number of messages across all processors.

We then applied our synchronization elimination scheme to the base version as well as the globally optimized version (C-opt) for each program. The results shown in Table 5 reveal that the algorithm is surprisingly successful in eliminating the redundant Synch messages. Except for two programs, the algorithm eliminates all synchronization messages from the base programs. When we look at the results for the programs that are optimized for communication prior to synchronization analysis, however, the picture somewhat changes. In C-Opt versions of Jacobi and stfrg, since the communication loop is reduced to one, no synchronization messages can be eliminated.

To sum up, our communication optimization algorithm eliminates $37.3 \%$ of the data messages and synchronization messages and reduces communication volume across all processors by $26 \%$. Our synchronization elimination algorithm eliminates $96.8 \%$ of the synchronization messages in the message vectorized programs and $74 \%$ of the synchronization messages in the globally optimized programs. 
Table 1: Dataflow sets and PENDING_OUT sets for the example in Figure 6(c).

\begin{tabular}{|c||c|c|}
\hline communication in & SendSet & Pending \\
\hline \hline 1 & $\{(1,0),(2,1),(3,2),(4,3),(5,4),(6,5),(7,6)\}$ & $\{(0,1),(1,2),(2,3),(3,4),(4,5),(5,6),(6,7)\}$ \\
2 & $\{(0,1),(1,2),(2,3)\}$ & $\{(1,0),(2,1),(3,2)\}$ \\
3 & $\{(3,4),(4,5),(5,6),(6,7)\}$ & $\{(4,3),(5,4),(6,5),(7,6)\}$ \\
& & \\
\hline
\end{tabular}

\begin{tabular}{|c||c|c|}
\hline PENDING_OUT for & iteration 1 & iteration 2 \\
\hline \hline 1 & $\{\{(0,1),(1,2),(2,3),(3,4),(4,5),(5,6),(6,7)\},\{\emptyset\},\{\emptyset\}\}$ & $\{\{\emptyset\},\{\emptyset\},\{\emptyset\}\}$ \\
2 & $\{\{(3,4),(4,5),(5,6),(6,7)\},\{\emptyset\},\{\emptyset\}\}$ & $\{\{\emptyset\},\{\emptyset\},\{\emptyset\}\}$ \\
3 & $\left\{\begin{array}{l}\{\emptyset\},\{\emptyset\},\{\emptyset\}\} \\
\{\emptyset\},\{\emptyset\},\{\emptyset\}\}\end{array}\right.$ \\
\hline
\end{tabular}

\section{Related Work}

Previously several methods have been presented to optimize the communication on distributed-memory message-passing machines. Most of the efforts considered communication optimization at loop (or array assignment statement) level. Although each approach has its own unique features, the general idea is to apply an appropriate combination of message vectorization, message coalescing and message aggregation $[9,3]$. Recently some researchers have proposed techniques for optimizing communication across multiple loop nests. The works in [5], [7], [12], [4], and [14] present similar frameworks to optimize send/recv communication globally and use variants of Regular Section Descriptors (RSD). Although this representation is convenient for simple array sections such as those found in block distributions, it is hard to embed alignment and general distribution information into it. Apart from this, working with section descriptors may result in overestimation of the communication sets. Instead, our approach is based on a linear algebra framework, and can represent all HPF-like alignment and distribution information accurately.

The approaches given in [6] and [8] examine the problem of eliminating redundant synchronization operations by piggy-backing them on data messages. Our approach is superior to both of these in eliminating synchronization as explained in the paper. Tseng [13] focuses on synchronization elimination problem. There is an important difference between our work and his. He eliminates synchronizations which are introduced by the insufficient communication analysis performed by the shared memory compilers. A compiler approach based on a distributed memory paradigm (like ours) does not insert those synchronizations in the first place. In our case, we start with an unoptimized program in which those types of artificial synchronizations are non-existent anyway. We rather focus on elimination of synchronizations that are caused by mandatory data communications. Such types of synchronization are not eliminated by Tseng's solution [13].

\section{Summary}

We presented dataflow algorithms to reduce number of data messages, communication volume and number of synchronization messages. Our experimental results revealed that our approach is quite successful in practice reducing on average $37.3 \%$ of data messages and $96.8 \%$ of synchronization messages in the message vectorized programs. We are working on compiling data-parallel programs with Get primitives, and elimination of synchronizations and possible deadlocks from programs compiled under hybrid approaches which employ both Put/Get and send/recv primitives.

\section{References}

[1] A. V. Aho, R. Sethi, and J. Ullman. Compilers: Principles, techniques, and tools. Addison-Wesley, 1986.

[2] A. Ancourt, F. Coelho, F. Irigoin, and R. Keryell. A linear algebra framework for static HPF code distribution. Scientific Programming, 6(1):3-28, Spring 1997.

[3] P. Banerjee, J. Chandy, M. Gupta, E. Hodges, J. Holm, A. Lain, D. Palermo, S. Ramaswamy, and E. Su. The PARADIGM compiler for distributed-memory multicomputers. IEEE Computer, 28(10):37-47, October 1995.

[4] S. Chakrabarti, M. Gupta, and J.-D. Choi. Global communication analysis and optimization. In Proc. ACM SIGPLAN Conference on Programming Language Design and Implementation, pages 68-78, Philadelphia, PA, May 1996.

[5] C. Gong, R. Gupta, and R. Melhem. Compilation techniques for optimizing communication on distributed-memory systems. In Proc. International Conference on Parallel Processing, Volume II, pages 39 46, St. Charles, IL, August 1993.

[6] M. Gupta, and E. Schonberg. Static analysis to reduce synchronization costs in data-parallel programs. In Proc. ACM Conference on Principles of Programming Languages, pages 322-332, St. Petersburg, FL, 1996.

[7] M. Gupta, E. Schonberg, and H. Srinivasan. A unified data-flow framework for optimizing communication. In Languages and Compilers for Parallel Computing, K. Pingali et al. (Eds.), Lecture Notes in Computer Science, Volume 892, pages 266-282, 1995.

[8] S. Hinrichs. Synchronization elimination in the deposit model. In Proc. 1996 International Conference on Parallel Processing, pages 87-94, St. Charles, IL, 1996.

[9] S. Hiranandani, K. Kennedy, and C. Tseng. Compiling Fortran D for MIMD distributed-memory machines. In Communications of the ACM, 35(8):66-80, August 1992.

[10] M. Kandemir, P. Banerjee, A. Choudhary, J. Ramanujam, and N Shenoy. A combined communication and synchronization optimization algorithm for one-way communication. Technical Report, CPDCTR-97-03, Northwestern University, October 1997.

[11] W. Kelly, V. Maslov, W. Pugh, E. Rosser, T. Shpeisman, and D. Wonnacott. The Omega Library interface guide. Technical Report CS-TR3445, CS Dept., University of Maryland, College Park, March 1995.

[12] K. Kennedy, and A. Sethi. A constrained-based communication placement framework, Technical Report CRPC-TR95515-S, CRPC, Rice University, 1995.

[13] C.-W. Tseng. Compiler optimizations for eliminating barrier synchronization. In Proc. the 5th ACM Symposium on Principles and Practice of Parallel programming (PPOPP'95), Santa Barbara, CA, July 1995.

[14] X. Yuan, R. Gupta, and R. Melhem. Demand-driven data flow analysis for communication optimization. Parallel Processing Letters, 7(4):359-370, December 1997. 
Table 2: Programs in our experiment set and their characteristics $(+=$ block, block-cyclic( 4$)$ or cyclic; $*=$ not distributed).

\begin{tabular}{|c||c||c||c||c||c||c|}
\hline PROGRAM & SUITE & LINES & ARRAYS & SIZE & DISTRIBUTION & DESCRIPTION \\
\hline \hline Jacobi & - & 25 & $2 \times 2 D$ & $1024 \times 1024$ & $(+, *)$ & Jacobi iteration \\
\hline 2D hydro & Livermore 18 & 38 & $9 \times 2 D$ & $512 \times 512$ & $(+, *)$ & 2D hydrodynamics \\
\hline ADI & Livermore 8 & 30 & $3 \times 3 D, 3 \times 1 D$ & $256 \times 256 \times 2$ & $(+, *, *)$ & ADI fragment \\
\hline vpenta & Spec92/NAS & 147 & $2 \times 3 D, 7 \times 2 D$ & $128 \times 128 \times 3$ & $(*,+, *)$ & pentadiagonal inversion \\
\hline SOR & - & 25 & $2 \times 2 D$ & $256 \times 256$ & $(+, *)$ & successive over-relaxation \\
\hline stfrg & Livermore 7 & 17 & $1 \times 1 D$ & 1024 & $(+)$ & state fragment equation \\
\hline tomcatv & Spec95 & 190 & $7 \times 2 D, 2 \times 1 D$ & $512 \times 512$ & $(+, *)$ & 2D mesh generation \\
\hline swim256 & Spec95 & 428 & $14 \times 2 D$ & $512 \times 512$ & $(+, *)$ & shallow water eqn. solver \\
\hline
\end{tabular}

Table 3: Communication volume and number of data messages in the base (message vectorized) programs for a single iteration of the time-step loop.

\begin{tabular}{|c|c|c|c|c|c|c|c|c|c|c|c|c|}
\hline \multirow{3}{*}{ PROGRAM } & \multicolumn{6}{|c|}{ comm. volume (in thousand elements) } & \multicolumn{6}{|c|}{ no. of data messages (Synch messages) } \\
\hline & \multicolumn{3}{|c|}{ No. of Processors $=8$} & \multicolumn{3}{|c|}{ No. of Processors $=32$} & \multicolumn{3}{|c|}{ No. of Processors $=8$} & \multicolumn{3}{|c|}{ No. of Processors $=32$} \\
\hline & BLOCK & B-CYC & CYCLIC & BLOCK & B-CYC & CYCLIC & BLOCK & B-CYC & CYCLIC & BLOCK & $\mathrm{B}-\mathrm{CYC}$ & CYCLIC \\
\hline Jacobi & 14.4 & 522.2 & 2088.9 & 63.3 & 522.2 & 2088.9 & 14 & 16 & 16 & 62 & 64 & 64 \\
\hline 2D hydro & 57.1 & 1036.3 & 4169.8 & 252.9 & 1036.3 & 4169.8 & 112 & 128 & 128 & 496 & 512 & 512 \\
\hline ADI & 10.7 & 96.8 & 387.1 & 47.2 & 96.8 & 387.1 & 42 & 48 & 48 & 186 & 192 & 192 \\
\hline vpenta & 343.2 & 343.2 & 343.2 & 1519.7 & 1519.7 & 1519.7 & 784 & 784 & 784 & 13888 & 13888 & 13888 \\
\hline SOR & 21.3 & 193.5 & 774.2 & 94.5 & 193.5 & 774.2 & 84 & 96 & 96 & 372 & 384 & 384 \\
\hline stfrg & 0.2 & 4.6 & 6.1 & 0.7 & 4.6 & 6.1 & 42 & 64 & 48 & 186 & 256 & 192 \\
\hline tomcatv & 57.1 & 1040 & 8323.2 & 252.9 & 2072.6 & 8323.2 & 112 & 128 & 128 & 496 & 1024 & 1024 \\
\hline swim256 & 57.6 & 914.4 & 3649.1 & 229 & 914.4 & 3649.1 & 128 & 142 & 142 & 464 & 478 & 478 \\
\hline
\end{tabular}

Table 4: Percentage (\%) improvement over base (message vectorized) in communication volume and number of data messages.

\begin{tabular}{|c|c|c|c|c|c|c|c|c|c|c|c|c|}
\hline \multirow{3}{*}{ PROGRAM } & \multicolumn{6}{|c|}{ \% improvement in comm. volume } & \multicolumn{6}{|c|}{ \% improvement in no. of data messages } \\
\hline & \multicolumn{3}{|c|}{ No. of Processors $=8$} & \multicolumn{3}{|c|}{ No. of Processors $=32$} & \multicolumn{3}{|c|}{ No. of Processors $=8$} & \multicolumn{3}{|c|}{ No. of Processors $=32$} \\
\hline & BLOCK & B-CYC & CYCLIC & BLOCK & B-CYC & CYCLIC & BLOCK & $\mathrm{B}-\mathrm{CYC}$ & CYCLIC & BLOCK & B-CYC & CYCLIC \\
\hline Jacobi & $0 \%$ & $0 \%$ & $0 \%$ & $0 \%$ & $0 \%$ & $0 \%$ & $0 \%$ & $0 \%$ & $0 \%$ & $0 \%$ & $0 \%$ & $0 \%$ \\
\hline 2D hydro & $50 \%$ & $50 \%$ & $50 \%$ & $50 \%$ & $50 \%$ & $50 \%$ & $50 \%$ & $51 \%$ & $50 \%$ & $50 \%$ & $50 \%$ & $51 \%$ \\
\hline ADI & $0 \%$ & $0 \%$ & $0 \%$ & $0 \%$ & $0 \%$ & $0 \%$ & $0 \%$ & $0 \%$ & $0 \%$ & $0 \%$ & $0 \%$ & $0 \%$ \\
\hline vpenta & $1 \%$ & $1 \%$ & $1 \%$ & $1 \%$ & $1 \%$ & $1 \%$ & $79 \%$ & $78 \%$ & $78 \%$ & $78 \%$ & $78 \%$ & $78 \%$ \\
\hline SOR & $0 \%$ & $0 \%$ & $0 \%$ & $0 \%$ & $0 \%$ & $0 \%$ & $0 \%$ & $0 \%$ & $0 \%$ & $0 \%$ & $0 \%$ & $0 \%$ \\
\hline stfrg & $71 \%$ & $64 \%$ & $0 \%$ & $71 \%$ & $64 \%$ & $0 \%$ & $83 \%$ & $74 \%$ & $0 \%$ & $81 \%$ & $73 \%$ & $0 \%$ \\
\hline tomcatv & $74 \%$ & $74 \%$ & $75 \%$ & $75 \%$ & $75 \%$ & $75 \%$ & $74 \%$ & $74 \%$ & $75 \%$ & $75 \%$ & $75 \%$ & $74 \%$ \\
\hline swim256 & $37 \%$ & $41 \%$ & $43 \%$ & $41 \%$ & $41 \%$ & $43 \%$ & $44 \%$ & $42 \%$ & $42 \%$ & $43 \%$ & $41 \%$ & $43 \%$ \\
\hline average & $29 \%$ & $29 \%$ & $21 \%$ & $30 \%$ & $29 \%$ & $21 \%$ & $41 \%$ & $40 \%$ & $31 \%$ & $41 \%$ & $40 \%$ & $31 \%$ \\
\hline
\end{tabular}

Table 5: Percentage (\%) improvement in number of synchronization messages obtained by our approach over the message vectorized (base) and globally optimized (C-Opt) versions.

\begin{tabular}{|c|c|c|c|c|c|c|c|c|c|c|c|c|}
\hline \multirow{3}{*}{ PROGRAM } & \multicolumn{6}{|c|}{ No. of processors $=8$} & \multicolumn{6}{|c|}{ No. of Processors $=32$} \\
\hline & \multicolumn{2}{|c|}{ BLOCK } & \multicolumn{2}{|c|}{ B-CYC } & \multicolumn{2}{|c|}{ CYCLIC } & \multicolumn{2}{|c|}{ BLOCK } & \multicolumn{2}{|c|}{$\mathrm{B}-\mathrm{CYC}$} & \multicolumn{2}{|c|}{ CYCLIC } \\
\hline & Base & $\mathrm{C}-0 \mathrm{pt}$ & Base & $\mathrm{C}-0 \mathrm{pt}$ & Base & C-0pt & Base & C-0pt & Base & C-0pt & Base & $\mathrm{C}-0 \mathrm{pt}$ \\
\hline Jacobi & $100 \%$ & $0 \%$ & $100 \%$ & $0 \%$ & $100 \%$ & $0 \%$ & $100 \%$ & $0 \%$ & $100 \%$ & $0 \%$ & $100 \%$ & $0 \%$ \\
\hline 2D hydro & $100 \%$ & $100 \%$ & $100 \%$ & $100 \%$ & $100 \%$ & $100 \%$ & $100 \%$ & $100 \%$ & $100 \%$ & $100 \%$ & $100 \%$ & $100 \%$ \\
\hline ADI & $100 \%$ & $100 \%$ & $100 \%$ & $100 \%$ & $100 \%$ & $100 \%$ & $100 \%$ & $100 \%$ & $100 \%$ & $100 \%$ & $100 \%$ & $100 \%$ \\
\hline vpenta & $100 \%$ & $100 \%$ & $100 \%$ & $100 \%$ & $100 \%$ & $100 \%$ & $100 \%$ & $100 \%$ & $100 \%$ & $100 \%$ & $100 \%$ & $100 \%$ \\
\hline SOR & $100 \%$ & $100 \%$ & $100 \%$ & $100 \%$ & $100 \%$ & $100 \%$ & $100 \%$ & $100 \%$ & $100 \%$ & $100 \%$ & $100 \%$ & $100 \%$ \\
\hline stfrg & $83 \%$ & $0 \%$ & $75 \%$ & $0 \%$ & $83 \%$ & $0 \%$ & $83 \%$ & $0 \%$ & $75 \%$ & $0 \%$ & $83 \%$ & $0 \%$ \\
\hline tomcatv & $100 \%$ & $100 \%$ & $100 \%$ & $100 \%$ & $100 \%$ & $100 \%$ & $100 \%$ & $100 \%$ & $100 \%$ & $100 \%$ & $100 \%$ & $100 \%$ \\
\hline swim256 & $94 \%$ & $89 \%$ & $93 \%$ & $89 \%$ & $93 \%$ & $89 \%$ & $93 \%$ & $88 \%$ & $93 \%$ & $88 \%$ & $93 \%$ & $88 \%$ \\
\hline average & $97 \%$ & $74 \%$ & $96 \%$ & $74 \%$ & $97 \%$ & $74 \%$ & $97 \%$ & $74 \%$ & $96 \%$ & $74 \%$ & $98 \%$ & $74 \%$ \\
\hline
\end{tabular}

